\title{
LOCAL RISK-MINIMIZATION FOR MULTIDIMENSIONAL ASSETS AND PAYMENT STREAMS
}

\author{
MARTIN SCHWEIZER \\ ETH Zürich, Departement Mathematik \\ ETH-Zentrum, HG G 51.2 \\ CH-8092 Zürich, Switzerland \\ E-mail: martin.schweizer@math.ethz.ch
}

\begin{abstract}
One of the earliest concepts for hedging and pricing in incomplete financial markets has been the quadratic criterion of local risk-minimization. However, definitions and theory have so far been established only for the case of a single (one-dimensional) risky asset. We extend the approach to a general multidimensional setting and prove that the basic martingale characterization result for locally risk-minimizing strategies still holds true. In comparison with existing literature, the self-contained presentation is more streamlined, and a number of earlier imposed technical conditions are no longer needed. As a minor extension, we show how payment streams (instead of final payoffs only) can be handled as well.
\end{abstract}

0. Introduction. Since its inception 20 years ago in [9], local risk-minimization has become a popular criterion for hedging and pricing in incomplete financial markets. It has been and is still being used in many different areas, including transaction costs, American options, insider models, credit and default risk, life insurance liabilities, etc. Indeed, a Google Scholar search in early August 2007 with the key phrase "local risk-minimization" (enclosed in quotation marks) returned well over 100 genuine hits. One reason for this popularity lies in the fact that although the definition of locally risk-minimizing strategies is rather technical, they can be computed very easily and fairly explicitly in quite general semimartingale models. For some background reading, we refer to the survey article [13].

A closer look at the existing literature reveals, somewhat surprisingly, that local riskminimization has up to now been defined and studied only for the case where the fi-

2000 Mathematics Subject Classification: Primary 91B28; Secondary 60G48, 60G35.

Key words and phrases: local risk-minimization, quadratic hedging, incomplete markets, locally risk-minimizing, Föllmer-Schweizer decomposition, payment streams, structure condition, mean-variance tradeoff.

It is a pleasure to thank Olga Bobrovnytska for several discussions on the topic of this paper, and Thomas Møller and Michèle Vanmaele for pointing out some related references.

The paper is in final form and no version of it will be published elsewhere. 
nancial market contains one single risky asset. In this paper, we generalize the approach to $\mathbb{R}^{d}$-valued asset price processes $X$ and show that one obtains the same martingale characterization of locally risk-minimizing strategies as for $d=1$. We also remove several technical restrictions imposed in the original formulation, and extend the results from European contingent claims to payment streams. The overall presentation is deliberately kept self-contained.

The paper is structured as follows. Section 1 introduces the setup, defines local riskminimization and formulates its equivalent characterization as the main result in Theorem 1.6. Section 2 contains two auxiliary results, and Section 3 presents a general convergence result for certain quantities appearing in our analysis. In Section 4, we prove the main result and comment on its relation to the existing literature, and Section 5 briefly presents the link to the Föllmer-Schweizer decomposition.

1. Setting, problem formulation and main result. This section explains the basic problem, introduces required terminology and concepts, and formulates our main result.

We start with a filtered probability space $(\Omega, \mathcal{F}, \mathbb{F}, P)$, where $T>0$ is a finite time horizon and the filtration $\left(\mathcal{F}_{t}\right)_{0 \leq t \leq T}$ satisfies the usual conditions. All processes are indexed by time $t$ with $0 \leq t \leq T$. Discounted asset prices are given by an $\mathbb{R}^{d}$-valued RCLL semimartingale $X=\left(X_{t}\right)_{0 \leq t \leq T}$, and we assume that $X$ satisfies the structure condition $(S C)$. This means that $X$ is special with canonical decomposition

$$
X=X_{0}+M+A=X_{0}+M+\int d\langle M\rangle \lambda,
$$

where $M$ is in $\mathcal{M}_{0, \text { loc }}^{2}$ and $\lambda$ is $\mathbb{R}^{d}$-valued, predictable and in $L_{\text {loc }}^{2}(M)$, so that the meanvariance tradeoff process $K:=\int \lambda d A=\int \lambda^{\operatorname{tr}} d\langle M\rangle \lambda$ satisfies $K_{T}<\infty P$-a.s. It is well known that (SC) is related to an absence-of-arbitrage condition; see [2] and [12].

Let $B$ be a bounded, strictly increasing, predictable (real-valued) process null at 0 such that $\left\langle M^{i}, M^{j}\right\rangle \ll B$ for all $i, j$. One example is $B_{t}:=\tanh \left(t+\sum_{i=1}^{d}\left\langle M^{i}\right\rangle_{t}\right)$, and we shall see later that the choice of $B$ does not affect our main result. We denote by $P_{B}:=P \otimes B$ the finite measure on $(\Omega \times[0, T], \mathcal{F} \otimes \mathcal{B}([0, T]))$ given by $P_{B}[D]:=E\left[\int_{0}^{T} I_{D}(\omega, s) d B_{s}(\omega)\right]$ and define the matrix-valued predictable process $\sigma$ by $d\langle M\rangle=\sigma d B$. Each $\sigma_{t}(\omega)$ is a nonnegative definite symmetric $d \times d$-matrix, and we note for future use that

$$
\left\langle\int \vartheta d M, \int \zeta d M\right\rangle=\int \vartheta^{\operatorname{tr}} d\langle M\rangle \zeta=\int \vartheta^{\operatorname{tr}} \sigma \zeta d B \quad \text { for } \vartheta, \zeta \in L_{\text {loc }}^{2}(M)
$$

and that hence $\int \delta^{\operatorname{tr}} \sigma \delta d B$ is bounded iff $\left\langle\int \delta d M\right\rangle$ is bounded. Moreover, we point out that $\int \delta d X$ is in $\mathcal{S}^{2}(P)$ (see below) for every $\mathbb{R}^{d}$-valued predictable process $\delta$ such that $\left\langle\int \delta d M\right\rangle$ and $\int|\delta d A|$ are both bounded. This will be important later when we define small perturbations.

Definition 1.1. The space $\Theta_{S}$ consists of all $\mathbb{R}^{d}$-valued predictable processes $\vartheta$ such that the stochastic integral process $\int \vartheta d X$ is well-defined and in the space $\mathcal{S}^{2}(P)$ of semimartingales. This means that

$$
E\left[\int_{0}^{T} \vartheta_{s}^{\operatorname{tr}} d\langle M\rangle_{s} \vartheta_{s}+\left(\int_{0}^{T}\left|\vartheta_{s} d A_{s}\right|\right)^{2}\right]<\infty
$$


or equivalently that $\int_{0}^{T} \vartheta_{s}^{\mathrm{tr}} \sigma_{s} \vartheta_{s} d B_{s}+\left(\int_{0}^{T}\left|\vartheta_{s}^{\mathrm{tr}} \sigma_{s} \lambda_{s}\right| d B_{s}\right)^{2} \in L^{1}(P)$. An $L^{2}$-strategy is a pair $\varphi=(\vartheta, \eta)$, where $\vartheta \in \Theta_{S}$ and $\eta$ is a real-valued adapted process such that the value process $V(\varphi):=\vartheta^{\operatorname{tr}} X+\eta$ is right-continuous and square-integrable, i.e., $V_{t}(\varphi) \in L^{2}(P)$ for all $t \in[0, T] . \varphi$ is called 0 -achieving if $V_{T}(\varphi)=0 P$-a.s.

As usual, a strategy $\varphi=(\vartheta, \eta)$ describes how we trade in the financial market given by $X$. At time $t$, we hold $\vartheta_{t}^{i}$ shares of asset $i$ for $i=1, \ldots, d$ and have the amount $\eta_{t}$ in a riskless bank account with zero interest rate and hence constant value 1 . We next consider a payment stream $H=\left(H_{t}\right)_{0 \leq t \leq T}$ kept fixed throughout the sequel. Mathematically, $H$ is right-continuous, adapted, real-valued and square-integrable; the interpretation is that $H_{t} \in L^{2}(P)$ represents the total payments on $[0, t]$ arising due to some financial contract. A European contingent claim with maturity $T$ would have $H_{t}=0$ for all $t<T$ and just an $\mathcal{F}_{T}$-measurable payoff $H_{T} \in L^{2}(P)$ due at time $T$; in general, the process $H$ involves both cash inflows and outlays, and can but need not be of finite variation. We want to hedge $H$ in a quadratic sense, and so first assign to each $L^{2}$-strategy a cost and a quadratic risk process.

Definition 1.2. Fix a payment stream $H$. The (cumulative) cost process of an $L^{2}-$ strategy $\varphi=(\vartheta, \eta)$ is

$$
C_{t}^{H}(\varphi):=H_{t}+V_{t}(\varphi)-\int_{0}^{t} \vartheta_{s} d X_{s}, \quad 0 \leq t \leq T .
$$

$\varphi$ is called self-financing (for $H$ ) if $C^{H}(\varphi)$ is constant, and mean-self-financing if $C^{H}(\varphi)$ is a martingale (which is then square-integrable). The risk process of $\varphi$ is

$$
R_{t}^{H}(\varphi):=E\left[\left(C_{T}^{H}(\varphi)-C_{t}^{H}(\varphi)\right)^{2} \mid \mathcal{F}_{t}\right], \quad 0 \leq t \leq T .
$$

As usual, $C_{t}^{H}(\varphi)$ describes the cumulative costs on $[0, t]$ from paying according to $H$ and trading according to $\varphi$; see [6] and [5]. One difference to the well-known situation in dimension $d=1$ and with a European contingent claim $H_{T}$ is that we use here a different attribution of value and costs; this was suggested in [7] and also used in [8] and [1]. Indeed, our total cost $C_{T}^{H}$ is the same as in the approach in [11], but we use strategies with $V_{T}=0$ instead of $V_{T}=H_{T}$. This is (with hindsight) better suited for an extension to payment streams.

REMARK 1.3. For later use, we observe that if $\varphi=(\vartheta, \eta)$ is a 0 -achieving and mean-selffinancing $L^{2}$-strategy for $H$, then $\varphi$ is uniquely determined from $\vartheta$ (and of course $H$ ). To see this, use the definition and martingale property of $C^{H}(\varphi)$ and $V_{T}(\varphi)=0$ to obtain

$$
\begin{aligned}
\eta_{t} & =V_{t}(\varphi)-\vartheta_{t}^{\operatorname{tr}} X_{t}=E\left[H_{T}-\int_{0}^{T} \vartheta_{s} d X_{s} \mid \mathcal{F}_{t}\right]-H_{t}+\int_{0}^{t} \vartheta_{s} d X_{s}-\vartheta_{t}^{\operatorname{tr}} X_{t} \\
& =E\left[H_{T}-H_{t}-\int_{t}^{T} \vartheta_{s} d X_{s} \mid \mathcal{F}_{t}\right]-\vartheta_{t}^{\operatorname{tr}} X_{t} .
\end{aligned}
$$

Clearly, $R^{H}(\varphi)$ is then determined by $\vartheta$ as well.

As in [11], we want to minimize $R^{H}(\varphi)$ with respect to small perturbations of $\varphi$, to be introduced next. A partition of $[0, T]$ is a set $\tau=\left\{t_{0}, t_{1}, \ldots, t_{k}\right\} \subseteq[0, T]$ with $0=t_{0}<t_{1}<\cdots<t_{k}=T$, and its mesh size is $|\tau|:=\max \left\{t_{i+1}-t_{i} \mid t_{i}, t_{i+1} \in \tau\right\}$. Note 
that $k$ may vary with $\tau$. A sequence $\left(\tau_{n}\right)_{n \in \mathbb{N}}$ of partitions is increasing if $\tau_{n} \subseteq \tau_{n+1}$ for all $n$; it tends to the identity if $\lim _{n \rightarrow \infty}\left|\tau_{n}\right|=0$. To each partition $\tau$, we associate on $\Omega \times[0, T]$ the $\sigma$-fields

$$
\begin{aligned}
& \mathcal{P}^{\tau}:=\sigma\left(\left\{D_{0} \times\{0\}, D_{i} \times\left(t_{i}, t_{i+1}\right] \mid t_{i}, t_{i+1} \in \tau, D_{0} \in \mathcal{F}_{0}, D_{i} \in \mathcal{F}_{t_{i}}\right\}\right), \\
& \mathcal{O}^{\tau}:=\sigma\left(\left\{D_{0} \times\{0\}, D_{i+1} \times\left(t_{i}, t_{i+1}\right] \mid t_{i}, t_{i+1} \in \tau, D_{0} \in \mathcal{F}_{0}, D_{i+1} \in \mathcal{F}_{t_{i+1}}\right\}\right) .
\end{aligned}
$$

One easily sees that $\sigma\left(\bigcup_{n \in \mathbb{N}} \mathcal{P}^{\tau_{n}}\right)$ equals the predictable $\sigma$-field $\mathcal{P}$ for any sequence of partitions tending to the identity; hence $\mathcal{P}^{\tau_{n}}$ increases to $\mathcal{P}$ if $\left(\tau_{n}\right)$ is in addition increasing.

Definition 1.4. A pair $\Delta=(\delta, \varepsilon)$ consisting of an $\mathbb{R}^{d}$-valued predictable process $\delta$ and an adapted real-valued process $\varepsilon$ is called a small perturbation if $\left\langle\int \delta d M\right\rangle=\int \delta^{\operatorname{tr}} \sigma \delta d B$ and $\left|\delta^{\operatorname{tr}} \sigma \lambda\right|$ (and hence also $\int|\delta d A|=\int\left|\delta^{\operatorname{tr}} \sigma \lambda\right| d B$ ) are bounded (uniformly in $t, \omega$ ), the process $V(\Delta):=\delta^{\operatorname{tr}} X+\varepsilon$ is square-integrable, and $V_{T}(\Delta)=0 P$-a.s. (Note that $\Delta$ need not be an $L^{2}$-strategy, although $\delta \in \Theta_{S}$ and $\Delta$ is 0 -achieving: We do not require that $V(\Delta)$ is right-continuous.) For each subinterval $(s, t]$ of $[0, T]$, we then define the small perturbation

$$
\left.\Delta\right|_{(s, t]}:= \begin{cases}\left(\delta I_{\rrbracket] s, t]}, \varepsilon I_{[\llbracket s, t \llbracket}\right) & \text { if } t<T, \\ \left(\delta I_{\rrbracket] s, T]]}, \varepsilon I_{[[s, T \rrbracket}\right) & \text { if } t=T .\end{cases}
$$

For an $L^{2}$-strategy $\varphi$, a small perturbation $\Delta$ and a partition $\tau$ of $[0, T]$, we set

$$
r^{\tau}[\varphi, \Delta ; H]:=\sum_{t_{i}, t_{i+1} \in \tau} \frac{R_{t_{i}}^{H}\left(\varphi+\left.\Delta\right|_{\left(t_{i}, t_{i+1}\right]}\right)-R_{t_{i}}^{H}(\varphi)}{E\left[B_{t_{i+1}}-B_{t_{i}} \mid \mathcal{F}_{t_{i}}\right]} I_{\left(t_{i}, t_{i+1}\right]} .
$$

Recall that the payment stream $H$ is fixed throughout. Note that $V(\psi), C^{H}(\psi), R^{H}(\psi)$ and hence $r^{\tau}[\varphi, \Delta ; H]$ are well-defined without any requirement of right-continuity for $V(\psi)$.

As in $[11], r^{\tau}[\varphi, \Delta ; H]$ is a measure for the increase of quadratic risk when $\varphi$ is perturbed locally by $\Delta$. Note that we have slightly modified the original definition of $\left.\Delta\right|_{(s, t]}$ in [11] to account for the special case $t=T$. This allows us to drop the assumption, imposed in [11], that $M$ is $P$-a.s. continuous at $T$, since we no longer require for a small perturbation that $\delta_{T}=0$. In fact, every $\mathbb{R}^{d}$-valued predictable process $\delta$ with $\left\langle\int \delta d M\right\rangle$ and $\left|\delta^{\operatorname{tr}} \sigma \lambda\right|$ both bounded can be extended to a small perturbation $\Delta=(\delta, \varepsilon)$, given by the 0 -achieving mean-self-financing $L^{2}$-strategy associated to $\delta$ by Remark 1.3.

The second (and important) difference to [11] is the denominator of $r^{\tau}[\varphi, \Delta ; H]$ in (1.3). For a vector-valued $M$, we cannot divide by the (perhaps non-invertible) matrix $E\left[\langle M\rangle_{t_{i+1}}-\langle M\rangle_{t_{i}} \mid \mathcal{F}_{t_{i}}\right]$; the appropriate "time scale" is now instead given by $B$. Our choice of $B$ as strictly increasing will also simplify some arguments later on.

Definition 1.5. Fix a payment stream $H$. An $L^{2}$-strategy $\varphi$ is called locally riskminimizing for $H$ if for every small perturbation $\Delta$ and every increasing sequence $\left(\tau_{n}\right)_{n \in \mathbb{N}}$ of partitions tending to the identity, we have

$$
\liminf _{n \rightarrow \infty} r^{\tau_{n}}[\varphi, \Delta ; H] \geq 0 \quad P_{B} \text {-a.e. }
$$

Our main result is now the following extension of Proposition 2.3 in [11], or Theorem 3.3 in [13]. 
THEOREM 1.6. Suppose the $\mathbb{R}^{d}$-valued semimartingale $X$ satisfies the structure condition $(S C)$ and let $H$ be a payment stream. If the mean-variance tradeoff process $K=$ $\int \lambda^{\operatorname{tr}} d\langle M\rangle \lambda$ (or, equivalently, A) is continuous, the following are equivalent for an $L^{2}$ strategy $\varphi$ :

1) $\varphi$ is locally risk-minimizing for $H$.

2) $\varphi$ is 0-achieving and mean-self-financing, and the cost process $C^{H}(\varphi)$ is strongly orthogonal to $M$.

In particular, the concept "locally risk-minimizing" does not depend on the choice of B.

We prove Theorem 1.6 in Section 4 where we also provide additional comments.

In our proof, we shall need small perturbations which satisfy the additional requirement that $\varepsilon_{t}=0$ for all $t<T$. The next auxiliary result shows how this can be achieved.

Lemma 1.7. Suppose $X$ satisfies the structure condition (SC). For any $\mathbb{R}^{d}$-valued predictable process $\delta$, there is a sequence $\Delta^{m}=\left(\delta^{m}, \varepsilon^{m}\right), m \in \mathbb{N}$, of small perturbations such that $\varepsilon_{t}^{m}=0$ for $t<T$ and each $m$, and $\lim _{m \rightarrow \infty} \delta^{m}=\delta P_{B}$-a.e. More precisely, $\delta^{m}$ has the form

$$
\delta^{m}=\delta I_{\left[0, \varrho_{m}\right]} I_{\{|\delta| \leq m\}} I_{\left\{\delta^{\operatorname{tr}} \sigma \delta \leq m\right\}} I_{\left\{\left|\delta^{\operatorname{tr}} \sigma \lambda\right| \leq m\right\}}
$$

for an increasing sequence of stopping times $\varrho_{m} \nearrow T P$-a.s.

Proof. By the structure condition $(\mathrm{SC}), X$ is in $\mathcal{S}_{\text {loc }}^{2}(P)$, and so there are stopping times $\varrho_{m} \nearrow T P$-a.s. with $X_{\varrho_{m}}^{*}:=\sup _{0 \leq t \leq \varrho_{m}}\left|X_{t}\right| \in L^{2}(P)$ for each $m$. Now define the predictable process $\delta^{m}$ by $(1.4)$ and $\Delta^{m}:=\left(\delta^{m}, \varepsilon^{m}\right)$ by

$$
\varepsilon_{t}^{m}:=-I_{\{T\}}(t)\left(\delta_{t}^{m}\right)^{\operatorname{tr}} X_{t} .
$$

Then $\varepsilon^{m}$ is adapted with $\varepsilon_{t}^{m}=0$ for all $t<T$, and $\left|\left(\delta^{m}\right)^{\operatorname{tr}} \sigma \lambda\right|$ and $\left\langle\int \delta^{m} d M\right\rangle$ are both bounded, since $B$ is bounded. Moreover, $V_{T}\left(\Delta^{m}\right)=0$ and for $t<T$, (1.4) gives $\left|V_{t}\left(\Delta^{m}\right)\right|=\left|\left(\delta_{t}^{m}\right)^{\operatorname{tr}} X_{t}\right| \leq m X_{\varrho_{m}}^{*} \in L^{2}(P)$ so that $\Delta^{m}$ is a small perturbation. Finally, (1.4) clearly implies $\delta^{m} \rightarrow \delta P_{B}$-a.e. as $m \rightarrow \infty$.

2. Preliminary results. This section prepares the ground by proving two auxiliary results. Although these are analogous to earlier work in [11], we provide full details since our definitions here are slightly different and the results are a bit more general. We first show that when searching for locally risk-minimizing $L^{2}$-strategies, we can restrict our attention to 0-achieving and mean-self-financing ones. This is a more general version of Lemma 2.1 in [11]; thanks to our choice of $B$, we do not need the assumption that $\langle M\rangle$ (for $d=1$ ) is $P$-a.s. strictly increasing on $[0, T]$.

Proposition 2.1. Suppose $X$ satisfies the structure condition (SC) and fix a payoff process $H$. Then any locally risk-minimizing $L^{2}$-strategy is 0 -achieving and mean-selffinancing.

Proof. Let $\varphi=(\vartheta, \eta)$ be locally risk-minimizing; then $\varphi$ is 0 -achieving by definition. Define $\hat{\varphi}=(\hat{\vartheta}, \hat{\eta})$ by $\hat{\vartheta} \equiv \vartheta$ and like in $(1.1)$

$$
\hat{\eta}_{t}:=\left[H_{T}-\int_{0}^{T} \vartheta_{s} d X_{s} \mid \mathcal{F}_{t}\right]-H_{t}+\int_{0}^{t} \vartheta_{s} d X_{s}-\vartheta_{t}^{\mathrm{tr}} X_{t}, \quad 0 \leq t \leq T,
$$


choosing a right-continuous version of the martingale given by the conditional expectations. Then $\hat{\varphi}$ is an $L^{2}$-strategy, because $\hat{\vartheta}$ is in $\Theta_{S}, \hat{\eta}$ is adapted, and $V(\hat{\varphi})$ is like $H$ and $\int \vartheta d X$ right-continuous and square-integrable. Moreover, $\hat{\varphi}$ is clearly 0 -achieving and by definition of $\hat{\eta}$ also mean-self-financing. Because $C_{T}^{H}(\hat{\varphi})=H_{T}-\int_{0}^{T} \vartheta_{s} d X_{s}=C_{T}^{H}(\varphi)$, we thus have

$$
C_{t}^{H}(\hat{\varphi})=E\left[C_{T}^{H}(\hat{\varphi}) \mid \mathcal{F}_{t}\right]=E\left[C_{T}^{H}(\varphi) \mid \mathcal{F}_{t}\right] \quad \text { for any } t \in[0, T] .
$$

Moreover, $\Delta:=\hat{\varphi}-\varphi=(0, \hat{\eta}-\eta)=:(\delta, \varepsilon)$ is like $\hat{\varphi}$ and $\varphi$ an $L^{2}$-strategy and 0 -achieving, and since $\delta \equiv 0, \Delta$ is even a small perturbation.

Now take any partition $\tau$ of $[0, T]$ and $t_{i}, t_{i+1} \in \tau$. Since $\left.\Delta\right|_{\left(t_{i}, t_{i+1}\right]}$ is like $\Delta$ a small perturbation and hence 0-achieving, we get $V_{T}\left(\varphi+\left.\Delta\right|_{\left(t_{i}, t_{i+1}\right]}\right)=0=V_{T}(\varphi)$ and thus

$$
C_{T}^{H}\left(\varphi+\left.\Delta\right|_{\left(t_{i}, t_{i+1}\right]}\right)=C_{T}^{H}(\varphi),
$$

since $\delta \equiv 0$. For $t_{i}<T$, we have by (1.2) and (2.1)

$$
\begin{aligned}
C_{t_{i}}^{H}\left(\varphi+\left.\Delta\right|_{\left(t_{i}, t_{i+1}\right]}\right) & =H_{t_{i}}+V_{t_{i}}\left(\varphi+\left.\Delta\right|_{\left(t_{i}, t_{i+1}\right]}\right)-\int_{0}^{t_{i}} \vartheta_{s} d X_{s} \\
& =H_{t_{i}}+\vartheta_{t_{i}}^{\mathrm{tr}} X_{t_{i}}+\hat{\eta}_{t_{i}}-\int_{0}^{t_{i}} \vartheta_{s} d X_{s} \\
& =C_{t_{i}}^{H}(\hat{\varphi})=E\left[C_{T}^{H}(\varphi) \mid \mathcal{F}_{t_{i}}\right] .
\end{aligned}
$$

Therefore we obtain by using (2.1)

$$
\begin{aligned}
R_{t_{i}}^{H}\left(\varphi+\left.\Delta\right|_{\left(t_{i}, t_{i+1}\right]}\right) & =E\left[\left(C_{T}^{H}(\varphi)-E\left[C_{T}^{H}(\varphi) \mid \mathcal{F}_{t_{i}}\right]\right)^{2} \mid \mathcal{F}_{t_{i}}\right] \\
& =E\left[\left(C_{T}^{H}(\varphi)-C_{t_{i}}^{H}(\varphi)\right)^{2} \mid \mathcal{F}_{t_{i}}\right]-\left(C_{t_{i}}^{H}(\varphi)-E\left[C_{T}^{H}(\varphi) \mid \mathcal{F}_{t_{i}}\right]\right)^{2} \\
& =R_{t_{i}}^{H}(\varphi)-\left(C_{t_{i}}^{H}(\varphi)-E\left[C_{T}^{H}(\varphi) \mid \mathcal{F}_{t_{i}}\right]\right)^{2}
\end{aligned}
$$

and hence

$$
\begin{aligned}
r^{\tau}[\varphi, \Delta ; H] & =\sum_{t_{i}, t_{i+1} \in \tau} \frac{R_{t_{i}}^{H}\left(\varphi+\left.\Delta\right|_{\left(t_{i}, t_{i+1}\right]}\right)-R_{t_{i}}^{H}(\varphi)}{E\left[B_{t_{i+1}}-B_{t_{i}} \mid \mathcal{F}_{t_{i}}\right]} I_{\left(t_{i}, t_{i+1}\right]} \\
& =-\sum_{t_{i}, t_{i+1} \in \tau} \frac{\left(C_{t_{i}}^{H}(\varphi)-E\left[C_{T}^{H}(\varphi) \mid \mathcal{F}_{t_{i}}\right]\right)^{2}}{E\left[B_{t_{i+1}}-B_{t_{i}} \mid \mathcal{F}_{t_{i}}\right]} I_{\left(t_{i}, t_{i+1}\right]} .
\end{aligned}
$$

This already indicates that for a non-mean-self-financing $L^{2}$-strategy, $r^{\tau}[\varphi, \Delta ; H]$ will probably become negative somewhere, and we now show that this persists asymptotically.

Since $\varphi$ is locally risk-minimizing, $\liminf _{n \rightarrow \infty} r^{\tau_{n}}[\varphi, \Delta ; H] \geq 0 P_{B}$-a.e. for every increasing sequence $\left(\tau_{n}\right)$ of partitions tending to the identity. Now take such a sequence and assume that for some $n_{0} \in \mathbb{N}$ and some $t_{0} \in \tau_{n_{0}}$, we have

$$
C_{t_{0}}^{H}(\varphi) \neq E\left[C_{T}^{H}(\varphi) \mid \mathcal{F}_{t_{0}}\right] \quad \text { on a set } \Gamma \in \mathcal{F} \text { with } P[\Gamma]>0 .
$$

Since we can and do choose both $C^{H}(\varphi)$ and $E\left[C_{T}^{H}(\varphi) \mid \mathbb{F}\right]$ right-continuous, there are for every $\omega \in \Gamma$ positive $\beta(\omega), \gamma(\omega)$ such that

$$
\left|\left(C_{t}^{H}(\varphi)-E\left[C_{T}^{H}(\varphi) \mid \mathcal{F}_{t}\right]\right)(\omega)\right| \geq \gamma(\omega)>0 \quad \text { for } \omega \in \Gamma \text { and every } t \in\left[t_{0}, t_{0}+2 \beta(\omega)\right] .
$$


Moreover, $B$ is bounded by some constant $k$, say; hence we can also choose versions of the conditional expectations such that for all $n \in \mathbb{N}$ and all $t_{i}, t_{i+1} \in \tau_{n}$,

$$
E\left[B_{t_{i+1}}-B_{t_{i}} \mid \mathcal{F}_{t_{i}}\right](\omega) \leq k \quad \text { for all } \omega \in \Omega .
$$

Now fix $\omega \in \Gamma$. Since $\left(\tau_{n}\right)$ is increasing and tends to the identity, we can find for every $t \in\left(t_{0}, t_{0}+\beta(\omega)\right]$ and all sufficiently large $n$ time points $t_{j}, t_{j+1} \in \tau_{n}$ with $t \in\left(t_{j}, t_{j+1}\right] \subseteq$ $\left(t_{0}, t_{0}+\beta(\omega)\right]$. This implies by $(2.2)$ that for such $t$,

$$
r^{\tau_{n}}[\varphi, \Delta ; H](t, \omega)=-\frac{\left(C_{t_{j}}^{H}(\varphi)-E\left[C_{T}^{H}(\varphi) \mid \mathcal{F}_{t_{j}}\right]\right)^{2}(\omega)}{E\left[B_{t_{j+1}}-B_{t_{j}} \mid \mathcal{F}_{t_{j}}\right](\omega)} \leq-\frac{\gamma^{2}(\omega)}{k}<0
$$

for all large enough $n$ and therefore

(2.3) $\liminf _{n \rightarrow \infty} r^{\tau_{n}}[\varphi, \Delta ; H](t, \omega) \leq-\frac{\gamma^{2}(\omega)}{k}<0 \quad$ for all $\omega \in \Gamma$ and $t \in\left(t_{0}, t_{0}+\beta(\omega)\right]$.

Because $B$ is strictly increasing and $P[\Gamma]>0,(2.3)$ yields

$$
P_{B}\left[\liminf _{n \rightarrow \infty} r^{\tau_{n}}[\varphi, \Delta ; H]<0\right] \geq E\left[I_{\Gamma}(\omega)\left(B_{t_{0}+\beta(\omega)}-B_{t_{0}}\right)(\omega)\right]>0
$$

which contradicts the assumption that $\varphi$ is locally risk-minimizing.

The above argument shows that if $\varphi$ is locally risk-minimizing, we have with probability 1 that $C_{t}^{H}(\varphi)=E\left[C_{T}^{H}(\varphi) \mid \mathcal{F}_{t}\right]$ simultaneously for all $t \in \bigcup_{n \in \mathbb{N}} \tau_{n}=: \mathcal{D}$. Since $\left(\tau_{n}\right)$ tends to the identity, $\mathcal{D}$ is dense in $[0, T]$, and so right-continuity of $C^{H}(\varphi)$ and $E\left[C_{T}^{H}(\varphi) \mid \mathbb{F}\right]$ yields that with probability $1, C_{t}^{H}(\varphi)=E\left[C_{T}^{H}(\varphi) \mid \mathcal{F}_{t}\right]$ for all $t \in[0, T]$. So $\varphi$ is indeed mean-self-financing.

Our second auxiliary result shows that we can decompose $r^{\tau}[\varphi, \Delta ; H]$ into a sum of four quantities. This will be useful later to analyze the asymptotic behaviour of $\left(r^{\tau_{n}}[\varphi, \Delta ; H]\right)_{n \in \mathbb{N}}$. Analogous results can be found in Section 2 of [11] and Section 3 of $[10]$.

Proposition 2.2. Assume that $X$ satisfies the structure condition (SC) and fix a payment stream $H$. For every 0 -achieving mean-self-financing $L^{2}$-strategy $\varphi=(\vartheta, \eta)$, every small perturbation $\Delta=(\delta, \varepsilon)$ and every partition $\tau$ of $[0, T]$, we then have

$$
r^{\tau}[\varphi, \Delta ; H]=A_{1}^{\tau}+A_{2}^{\tau}+A_{3}^{\tau}+A_{4}^{\tau}
$$

where

$$
\begin{aligned}
& A_{1}^{\tau}=E_{B}\left[\left(\delta-2 \mu^{H}\right)^{\operatorname{tr}} \sigma \delta \mid \mathcal{P}^{\tau}\right] \\
& A_{2}^{\tau}=\sum_{t_{i}, t_{i+1} \in \tau} \frac{\operatorname{Var}\left[\int_{t_{i}}^{t_{i+1}} \delta_{s} d A_{s} \mid \mathcal{F}_{t_{i}}\right]}{E\left[B_{t_{i+1}}-B_{t_{i}} \mid \mathcal{F}_{t_{i}}\right]} I_{\left(t_{i}, t_{i+1}\right]}, \\
& A_{3}^{\tau}=\sum_{t_{i}, t_{i+1} \in \tau} \frac{\operatorname{Cov}\left(\int_{t_{i}}^{t_{i+1}} \delta_{s} d M_{s}-\left(C_{t_{i+1}}^{H}(\varphi)-C_{t_{i}}^{H}(\varphi)\right), \int_{t_{i}}^{t_{i+1}} \delta_{s} d A_{s} \mid \mathcal{F}_{t_{i}}\right)}{E\left[B_{t_{i+1}}-B_{t_{i}} \mid \mathcal{F}_{t_{i}}\right]} I_{\left(t_{i}, t_{i+1}\right]}, \\
& A_{4}^{\tau}=\sum_{t_{i}, t_{i+1} \in \tau} \frac{\left(E\left[\int_{t_{i}}^{t_{i+1}} \delta_{s} d A_{s} \mid \mathcal{F}_{t_{i}}\right]+\varepsilon_{t_{i}}\right)^{2}}{E\left[B_{t_{i+1}}-B_{t_{i}} \mid \mathcal{F}_{t_{i}}\right]} I_{\left(t_{i}, t_{i+1}\right]},
\end{aligned}
$$


and $\mu^{H}$ is the integrand from the Galtchouk-Kunita-Watanabe decomposition of the martingale $C^{H}(\varphi)$ with respect to $M$.

Proof. For any small perturbation $\Delta^{\prime}=\left(\delta^{\prime}, \varepsilon^{\prime}\right)$, we denote by $C^{H}\left(\vartheta+\delta^{\prime}\right)$ the cost process of the unique 0 -achieving mean-self-financing $L^{2}$-strategy determined by $\vartheta+\delta^{\prime}$; see Remark 1.3 or the construction as in (1.1) of $\hat{\varphi}$ in the proof of Proposition 2.1. Since $\varphi$ itself is mean-self-financing, $C^{H}(\varphi)=C^{H}(\vartheta)$. Just plugging in the definitions gives

$$
C_{T}^{H}\left(\varphi+\left.\Delta\right|_{\left(t_{i}, t_{i+1}\right]}\right)=C_{T}^{H}(\varphi)-\int_{t_{i}}^{t_{i+1}} \delta_{s} d X_{s}=C_{T}^{H}\left(\vartheta+\delta I_{]] t_{i}, t_{i+1} \rrbracket}\right)
$$

and for $t_{i}<T$, by using the definitions and (2.4),

$$
\begin{aligned}
& C_{t_{i}}^{H}\left(\varphi+\left.\Delta\right|_{\left(t_{i}, t_{i+1}\right]}\right)=H_{t_{i}}+\vartheta_{t_{i}}^{\mathrm{tr}} X_{t_{i}}+\eta_{t_{i}}+\varepsilon_{t_{i}}-\int_{0}^{t_{i}} \vartheta_{s} d X_{s}=C_{t_{i}}^{H}(\varphi)+\varepsilon_{t_{i}}, \\
& C_{t_{i}}^{H}\left(\vartheta+\delta I_{\left.\rrbracket t_{i}, t_{i+1}\right]}\right)=E\left[C_{T}^{H}\left(\vartheta+\delta I_{\rrbracket t_{i}, t_{i+1} \rrbracket}\right) \mid \mathcal{F}_{t_{i}}\right]=C_{t_{i}}^{H}(\varphi)-E\left[\int_{t_{i}}^{t_{i+1}} \delta_{s} d A_{s} \mid \mathcal{F}_{t_{i}}\right] .
\end{aligned}
$$

Combining the above with the martingale property of $C^{H}\left(\vartheta+\delta I_{\rrbracket t_{i}, t_{i+1} \rrbracket}\right)$ yields

$$
\begin{aligned}
& =E\left[\left(C_{T}^{H}\left(\vartheta+\delta I_{\rrbracket t_{i}, t_{i+1} \rrbracket}\right)-C_{t_{i}}^{H}\left(\vartheta+\delta I_{\rrbracket t_{i}, t_{i+1} \rrbracket}\right)-\varepsilon_{t_{i}}-E\left[\int_{t_{i}}^{t_{i+1}} \delta_{s} d A_{s} \mid \mathcal{F}_{t_{i}}\right]\right)^{2} \mid \mathcal{F}_{t_{i}}\right] \\
& =R_{t_{i}}^{H}\left(\vartheta+\delta I_{\rrbracket t_{i}, t_{i+1} \rrbracket}\right)+\left(\varepsilon_{t_{i}}+E\left[\int_{t_{i}}^{t_{i+1}} \delta_{s} d A_{s} \mid \mathcal{F}_{t_{i}}\right]\right)^{2} \\
& =\operatorname{Var}\left[C_{T}^{H}\left(\vartheta+\delta I_{\rrbracket t_{i}, t_{i+1} \rrbracket}\right) \mid \mathcal{F}_{t_{i}}\right]+\left(\varepsilon_{t_{i}}+E\left[\int_{t_{i}}^{t_{i+1}} \delta_{s} d A_{s} \mid \mathcal{F}_{t_{i}}\right]\right)^{2} .
\end{aligned}
$$

Using (2.4) and $X=X_{0}+M+A$ now gives

$$
\begin{aligned}
\operatorname{Var}\left[C_{T}^{H}\left(\vartheta+\delta I_{\left.\rrbracket t_{i}, t_{i+1}\right]}\right) \mid \mathcal{F}_{t_{i}}\right] \\
=\operatorname{Var}\left[\int_{t_{i}}^{t_{i+1}} \delta_{s} d A_{s} \mid \mathcal{F}_{t_{i}}\right]+\operatorname{Var}\left[C_{T}^{H}(\vartheta)-\int_{t_{i}}^{t_{i+1}} \delta_{s} d M_{s} \mid \mathcal{F}_{t_{i}}\right] \\
-2 \operatorname{Cov}\left(C_{T}^{H}(\vartheta)-\int_{t_{i}}^{t_{i+1}} \delta_{s} d M_{s}, \int_{t_{i}}^{t_{i+1}} \delta_{s} d A_{s} \mid \mathcal{F}_{t_{i}}\right)
\end{aligned}
$$

Since $C^{H}(\vartheta)=C^{H}(\varphi)$ and $\int \delta d M$ are both martingales and $\int_{t_{i}}^{t_{i+1}} \delta_{s} d A_{s}$ is $\mathcal{F}_{t_{i+1}}$-measurable, the last term in (2.6) equals

$$
+2 \operatorname{Cov}\left(\int_{t_{i}}^{t_{i+1}} \delta_{s} d M_{s}-\left(C_{t_{i+1}}^{H}(\varphi)-C_{t_{i}}^{H}(\varphi)\right), \int_{t_{i}}^{t_{i+1}} \delta_{s} d A_{s} \mid \mathcal{F}_{t_{i}}\right) .
$$

Moreover, $C^{H}(\varphi)=C_{0}^{H}(\varphi)+\int \mu^{H} d M+L^{H}$ is the Galtchouk-Kunita-Watanabe decomposition of $C^{H}(\varphi)$ and so 


$$
\begin{aligned}
\operatorname{Var} & {\left[C_{T}^{H}(\vartheta)-\int_{t_{i}}^{t_{i+1}} \delta_{s} d M_{s} \mid \mathcal{F}_{t_{i}}\right] } \\
& =\operatorname{Var}\left[C_{t_{i+1}}^{H}(\varphi)-C_{t_{i}}^{H}(\varphi)-\int_{t_{i}}^{t_{i+1}} \delta_{s} d M_{s} \mid \mathcal{F}_{t_{i}}\right] \\
& =R_{t_{i}}^{H}(\varphi)+\operatorname{Cov}\left(\int_{t_{i}}^{t_{i+1}} \delta_{s} d M_{s}, \int_{t_{i}}^{t_{i+1}} \delta_{s} d M_{s}-2\left(C_{t_{i+1}}^{H}(\varphi)-C_{t_{i}}^{H}(\varphi)\right) \mid \mathcal{F}_{t_{i}}\right) \\
& =R_{t_{i}}^{H}(\varphi)+E\left[\int_{t_{i}}^{t_{i+1}} d\left\langle\int \delta d M, \int \delta d M-2 C^{H}(\varphi)\right\rangle_{s} \mid \mathcal{F}_{t_{i}}\right] \\
& =R_{t_{i}}^{H}(\varphi)+E\left[\int_{t_{i}}^{t_{i+1}} \delta_{s}^{\operatorname{tr}} d\langle M\rangle_{s}\left(\delta_{s}-2 \mu_{s}^{H}\right) \mid \mathcal{F}_{t_{i}}\right] .
\end{aligned}
$$

Now we combine (2.5)-(2.7) to obtain

$$
\begin{aligned}
R_{t_{i}}^{H}(\varphi+ & \left.\left.\Delta\right|_{\left(t_{i}, t_{i+1}\right]}\right)-R_{t_{i}}^{H}(\varphi) \\
= & E\left[\int_{t_{i}}^{t_{i+1}}\left(\delta_{s}-2 \mu_{s}^{H}\right)^{\operatorname{tr}} d\langle M\rangle_{s} \delta_{s} \mid \mathcal{F}_{t_{i}}\right]+\operatorname{Var}\left[\int_{t_{i}}^{t_{i+1}} \delta_{s} d A_{s} \mid \mathcal{F}_{t_{i}}\right] \\
& +2 \operatorname{Cov}\left(\int_{t_{i}}^{t_{i+1}} \delta_{s} d M_{s}-\left(C_{t_{i+1}}^{H}(\varphi)-C_{t_{i}}^{H}(\varphi)\right), \int_{t_{i}}^{t_{i+1}} \delta_{s} d A_{s} \mid \mathcal{F}_{t_{i}}\right) \\
& +\left(E\left[\int_{t_{i}}^{t_{i+1}} \delta_{s} d A_{s} \mid \mathcal{F}_{t_{i}}\right]+\varepsilon_{t_{i}}\right)^{2} .
\end{aligned}
$$

Dividing by $E\left[B_{t_{i+1}}-B_{t_{i}} \mid \mathcal{F}_{t_{i}}\right]$, multiplying by $I_{\left(t_{i}, t_{i+1}\right]}$ and summing over $t_{i}, t_{i+1} \in \tau$ gives $r^{\tau}[\varphi, \Delta ; H]$ on the left-hand side and the terms $A_{2}^{\tau}, A_{3}^{\tau}, A_{4}^{\tau}$ on the right-hand side. Moreover, we also obtain on the right-hand side the term

$$
\begin{aligned}
& \sum_{t_{i}, t_{i+1} \in \tau} \frac{E\left[\int_{t_{i}}^{t_{i+1}}\left(\delta_{s}-2 \mu_{s}^{H}\right)^{\operatorname{tr}} d\langle M\rangle_{s} \delta_{s} \mid \mathcal{F}_{t_{i}}\right]}{E\left[B_{t_{i+1}}-B_{t_{i}} \mid \mathcal{F}_{t_{i}}\right]} I_{\left(t_{i}, t_{i+1}\right]} \\
= & \sum_{t_{i}, t_{i+1} \in \tau} \frac{E\left[\int_{t_{i}}^{t_{i+1}}\left(\delta_{s}-2 \mu_{s}^{H}\right)^{\operatorname{tr}} \sigma_{s} \delta_{s} d B_{s} \mid \mathcal{F}_{t_{i}}\right]}{E\left[B_{t_{i+1}}-B_{t_{i}} \mid \mathcal{F}_{t_{i}}\right]} I_{\left(t_{i}, t_{i+1}\right]},
\end{aligned}
$$

and since this equals $E_{B}\left[\left(\delta-2 \mu^{H}\right)^{\operatorname{tr}} \sigma \delta \mid \mathcal{P}^{\tau}\right]=A_{1}^{\tau}$, the proof is complete.

3. Convergence results. We have seen in Proposition 2.2 that $r^{\tau}[\varphi, \Delta ; H]$ can be split into terms $A_{i}^{\tau}$ with $i=1, \ldots, 4$. This section studies the asymptotic behaviour of $\left(A_{i}^{\tau_{n}}\right)_{n \in \mathbb{N}}$ along an increasing sequence of partitions $\left(\tau_{n}\right)$ tending to the identity. The results are very similar to those in [10].

For this section, we introduce for brevity the following standing assumptions:

(3.1) $X$ satisfies the structure condition (SC);

$H$ is a payment stream;

$\varphi=(\vartheta, \eta)$ is a 0 -achieving and mean-self-financing $L^{2}$-strategy;

$\Delta=(\delta, \varepsilon)$ is a small perturbation;

$\left(\tau_{n}\right)_{n \in \mathbb{N}}$ is an increasing sequence of partitions of $[0, T]$ tending to the identity. 
Additional conditions will be added where needed. As in Section $2, \mu^{H}$ is the integrand in the Galtchouk-Kunita-Watanabe decomposition of $C^{H}(\varphi)$ with respect to $M$, and we write

$$
r^{\tau_{n}}[\varphi, \Delta ; H]=A_{1}^{n}+A_{2}^{n}+A_{3}^{n}+A_{4}^{n}
$$

for each $n \in \mathbb{N}$ by Proposition 2.2, using $A_{i}^{n}$ as shorthand for $A_{i}^{\tau_{n}}$.

LEMMA 3.1. Under the standing assumptions (3.1),

$$
\lim _{n \rightarrow \infty} A_{1}^{n}=\left(\delta-2 \mu^{H}\right)^{\operatorname{tr}} \sigma \delta \quad P_{B} \text {-a.e. }
$$

Proof. By Proposition 2.2, $A_{1}^{n}=E_{B}\left[\left(\delta-2 \mu^{H}\right)^{\operatorname{tr}} \sigma \delta \mid \mathcal{P}^{\tau_{n}}\right]$, and as observed in Section 1, $\mathcal{P}^{\tau_{n}}$ increases to the predictable $\sigma$-field $\mathcal{P}$ since $\left(\tau_{n}\right)$ is increasing and tends to the identity. Moreover, $\left(\delta-2 \mu^{H}\right)^{\operatorname{tr}} \sigma \delta$ is predictable and in $L^{1}\left(P_{B}\right)$ by the Cauchy-Schwarz inequality, since $\mu^{H}$ is in $\Theta_{S}$ and $\left\langle\int \delta d M\right\rangle$ is bounded. Hence (3.2) follows directly from the martingale convergence theorem.

It will later be important to know when the limit in (3.2) is nonnegative. The next result, a multidimensional extension of Proposition 1.1 in [10], settles this; note that its last condition on $\delta$ is satisfied whenever $\delta$ comes from a small perturbation $\Delta=(\delta, \varepsilon)$.

Proposition 3.2. Assume (3.1) and fix $\mu \in \Theta_{S}$. If $\mu^{\mathrm{tr}} \sigma \mu=0 P_{B}$-a.e., then for every $\delta \in \Theta_{S}$,

$$
(\delta-2 \mu)^{\operatorname{tr}} \sigma \delta \geq 0 \quad P_{B} \text {-a.e. }
$$

Conversely, if (3.3) holds for every $\mathbb{R}^{n}$-valued predictable $\delta$ such that $\left\langle\int \delta d M\right\rangle$ and $\left|\delta^{\operatorname{tr}} \sigma \lambda\right|$ are both bounded, then $\mu^{\mathrm{tr}} \sigma \mu=0 P_{B}$-a.e.

Proof. If $\mu^{\mathrm{tr}} \sigma \mu=0$, also $\mu^{\mathrm{tr}} \sigma \delta=0$ by the Cauchy-Schwarz inequality, and so (3.3) follows. Conversely, if (3.3) were valid for every $\delta \in \Theta_{S}$, we could choose $\delta:=\mu$ and immediately obtain $\mu^{\operatorname{tr}} \sigma \mu \leq 0$ so that $\mu^{\operatorname{tr}} \sigma \mu=0$ must hold since $\sigma$ is nonnegative definite. In the general case where (3.3) holds only for the smaller class of $\delta$ as in the statement, we first define

$$
\delta_{*}:=\mu I_{\left\{\mu^{\operatorname{tr}} \sigma \mu>0\right\}} \frac{\min \left(1,\left(\mu^{\operatorname{tr}} \sigma \mu\right)^{\frac{1}{2}}\right)}{\left(\mu^{\operatorname{tr}} \sigma \mu\right)^{\frac{1}{2}}} .
$$

Then $\delta_{*}$ is clearly $\mathbb{R}^{n}$-valued and predictable, and $\left\langle\int \delta_{*} d M\right\rangle=\int \delta_{*}^{\operatorname{tr}} \sigma \delta_{*} d B$ is bounded since $B$ is bounded and $0 \leq \delta_{*}^{\operatorname{tr}} \sigma \delta_{*} \leq 1$. Moreover, we have on the set $\left\{\mu^{\operatorname{tr}} \sigma \mu>1\right\}$ that

$$
\left(\delta_{*}-2 \mu\right)^{\operatorname{tr}} \sigma \delta_{*}=\left(\mu \frac{1}{\left(\mu^{\operatorname{tr}} \sigma \mu\right)^{\frac{1}{2}}}-2 \mu\right)^{\operatorname{tr}} \sigma \mu \frac{1}{\left(\mu^{\operatorname{tr}} \sigma \mu\right)^{\frac{1}{2}}}=1-2\left(\mu^{\operatorname{tr}} \sigma \mu\right)^{\frac{1}{2}}<-1,
$$

while on the set $\left\{0<\mu^{\operatorname{tr}} \sigma \mu \leq 1\right\}$, we have

$$
\left(\delta_{*}-2 \mu\right)^{\operatorname{tr}} \sigma \delta_{*}=(\mu-2 \mu)^{\operatorname{tr}} \sigma \mu=-\mu^{\operatorname{tr}} \sigma \mu<0 .
$$

Since $\mu^{\mathrm{tr}} \sigma \mu \geq 0 P_{B}$-a.e., the above shows that

$$
\left(\delta_{*}-2 \mu\right)^{\operatorname{tr}} \sigma \delta_{*}<0 \quad P_{B^{-}} \text {a.e. on }\left\{\mu^{\operatorname{tr}} \sigma \mu>0\right\} .
$$


Now let $\Delta^{m}=\left(\delta^{m}, \varepsilon^{m}\right), m \in \mathbb{N}$, be the sequence of small perturbations constructed from $\delta_{*}$ via Lemma 1.7. Then $\delta^{m}=\delta_{*} I_{\left[0, \varrho_{m}\right]} I_{\left\{\left|\delta_{*}\right| \leq m\right\}} I_{\left\{\delta_{*}^{\text {tr }} \sigma \delta_{*} \leq m\right\}} I_{\left\{\left|\delta_{*}^{\operatorname{tr}} \sigma \lambda\right| \leq m\right\}}$ and therefore

$$
\begin{aligned}
\left(\delta^{m}-2 \mu\right)^{\operatorname{tr}} \sigma \delta^{m}= & \left(\delta_{*}-2 \mu\right)^{\operatorname{tr}} \sigma \delta_{*} I_{\left[\left[0, \varrho_{m}\right]\right]} I_{\left\{\left|\delta_{*}\right| \leq m\right\}} I_{\left\{\delta_{*}^{\operatorname{tr}} \sigma \delta_{*} \leq m\right\}} I_{\left\{\left|\delta_{*}^{\operatorname{tr}} \sigma \lambda\right| \leq m\right\}} \\
& \rightarrow\left(\delta_{*}-2 \mu\right)^{\operatorname{tr}} \sigma \delta_{*} \quad P_{B^{-} \text {-a.e. as } m \rightarrow \infty}
\end{aligned}
$$

since $\varrho_{m} \nearrow T P$-a.s. By assumption, $\left(\delta^{m}-2 \mu\right)^{\operatorname{tr}} \sigma \delta^{m} \geq 0 P_{B}$-a.e. for every $m$, and therefore $\left(\delta_{*}-2 \mu\right)^{\operatorname{tr}} \sigma \delta_{*} \geq 0 P_{B}$-a.e. In view of (3.4), we must thus have $\mu^{\operatorname{tr}} \sigma \mu=0$ $P_{B}$-a.e.

Remark 3.3. Combining Lemma 3.1 and Proposition 3.2 shows that $\lim \inf _{n \rightarrow \infty} A_{1}^{n} \geq 0$ holds $P_{B}$-a.e. for every small perturbation if and only if $\left(\mu^{H}\right)^{\operatorname{tr}} \sigma \mu^{H}=0 P_{B}$-a.e. Equivalently, this says that $\left\langle\int \mu^{H} d M\right\rangle \equiv 0$, or $\int \mu^{H} d M \equiv 0$, which means that $C^{H}(\varphi)$ is strongly orthogonal to $M$. This is one of the two key ingredients for the proof of Theorem 1.6.

The second key ingredient in the proof of Theorem 1.6 is to show that the terms $A_{2}^{n}, A_{3}^{n}, A_{4}^{n}$ are all asymptotically negligible. This will be achieved by combining an estimate with a general convergence result, and we now proceed to develop the latter. This is a slight generalization of Lemma 2.1 in [10].

As in (3.1), let $\left(\tau_{n}\right)_{n \in \mathbb{N}}$ be an increasing sequence of partitions of $[0, T]$ tending to the identity. Let $Y=\left(Y_{t}\right)_{0 \leq t \leq T}$ be an adapted real-valued process with $Y_{0}=0$. For $p>0$ and $t \in[0, T]$, the $p$-variation of $Y$ on $[0, t]$ along $\left(\tau_{n}\right)$ is

$$
W_{p}(Y, t):=\sup _{n \in \mathbb{N}} \sum_{t_{i}, t_{i+1} \in \tau_{n}}\left|Y_{t_{i+1} \wedge t}-Y_{t_{i} \wedge t}\right|^{p}
$$

For any partition $\tau$ of $[0, T]$, we also define the processes

$$
\begin{aligned}
& Q_{p}[Y, \tau](\omega, t):=\sum_{t_{i}, t_{i+1} \in \tau} \frac{\left|Y_{t_{i+1}}-Y_{t_{i}}\right|^{p}}{B_{t_{i+1}}-B_{t_{i}}}(\omega) I_{\left(t_{i}, t_{i+1}\right]}(t), \\
& \tilde{Q}_{p}[Y, \tau](\omega, t):=\sum_{t_{i}, t_{i+1} \in \tau} \frac{E\left[\left|Y_{t_{i+1}}-Y_{t_{i}}\right|^{p} \mid \mathcal{F}_{t_{i}}\right]}{E\left[B_{t_{i+1}}-B_{t_{i}} \mid \mathcal{F}_{t_{i}}\right]}(\omega) I_{\left(t_{i}, t_{i+1}\right]}(t) .
\end{aligned}
$$

Both are nonnegative and well-defined since $B$ is strictly increasing; $Q_{p}[Y, \tau]$ is $\mathcal{O}^{\tau}$-measurable, and like at the end of Section 2, one readily verifies that

$$
\tilde{Q}_{p}[Y, \tau]=E_{B}\left[Q_{p}[Y, \tau] \mid \mathcal{P}^{\tau}\right]
$$

If $Y$ is increasing and $Y_{T}$ is integrable, $P_{Y}=P \otimes Y$ denotes as in Section 1 the finite measure induced by $P$ and $Y$ on $(\Omega \times[0, T], \mathcal{F} \otimes \mathcal{B}([0, T]))$.

LEMMA 3.4. If $Y$ is adapted, null at 0, increasing and $Y_{T}$ is integrable, then

$$
Q_{1}[Y, \tau]=\left.\frac{d P_{Y}}{d P_{B}}\right|_{\mathcal{O}^{\tau}} \quad \text { and } \quad \tilde{Q}_{1}[Y, \tau]=\left.\frac{d P_{Y}}{d P_{B}}\right|_{\mathcal{P}^{\tau}}
$$

for every partition $\tau$ of $[0, T]$.

Proof. Since $B$ is strictly increasing, $P_{Y} \ll P_{B}$. For any $t_{i}, t_{i+1} \in \tau$ and $D_{i+1} \in \mathcal{F}_{t_{i+1}}$,

$$
E_{B}\left[Q_{1}[Y, \tau] I_{D_{i+1} \times\left(t_{i}, t_{i+1}\right]}\right]=E\left[\frac{Y_{t_{i+1}}-Y_{t_{i}}}{B_{t_{i+1}}-B_{t_{i}}}\left(B_{t_{i+1}}-B_{t_{i}}\right) I_{D_{i+1}}\right]=E_{Y}\left[I_{D_{i+1} \times\left(t_{i}, t_{i+1}\right]}\right]
$$

so that $\left.\frac{d P_{Y}}{d P_{B}}\right|_{\mathcal{O}^{\tau}}=Q_{1}[Y, \tau]$. The second assertion immediately follows from (3.5). 
Proposition 3.5. Assume (3.1) and let $Y$ be an adapted process with $Y_{0}=0$. Let $1 \leq r<p$ and suppose $Y$ has integrable $r$-variation along $\left(\tau_{n}\right)$. If $Y$ is continuous, then

$$
\lim _{n \rightarrow \infty} Q_{p}\left[Y, \tau_{n}\right]=0 \quad P_{B} \text {-a.e. }
$$

If in addition

$$
\sup _{n \in \mathbb{N}} Q_{p}\left[Y, \tau_{n}\right] \in L^{1}\left(P_{B}\right)
$$

then we also have

$$
\lim _{n \rightarrow \infty} \tilde{Q}_{p}\left[Y, \tau_{n}\right]=0 \quad P_{B} \text {-a.e. }
$$

Proof. We first note that since $p>r$,

$$
Q_{p}\left[Y, \tau_{n}\right] \leq Q_{r}\left[Y, \tau_{n}\right] \sup _{t_{i}, t_{i+1} \in \tau_{n}}\left|Y_{t_{i+1}}-Y_{t_{i}}\right|^{p-r},
$$

and the second factor converges to 0 since $Y$ is $P$-a.s. uniformly continuous on $[0, T]$. Hence it is enough for (3.6) to show that $\sup _{n \in \mathbb{N}} Q_{r}\left[Y, \tau_{n}\right]<\infty P_{B}$-a.e. But if $U$ denotes the $r$-variation of $Y$ along $\left(\tau_{n}\right)$, we clearly have $\left|Y_{t_{i+1}}-Y_{t_{i}}\right|^{r} \leq U_{t_{i+1}}-U_{t_{i}}$ and thus by Lemma 3.4

$$
Q_{r}\left[Y, \tau_{n}\right] \leq Q_{1}\left[U, \tau_{n}\right]=\left.\frac{d P_{U}}{d P_{B}}\right|_{\mathcal{O}^{\tau_{n}}} .
$$

The last expression is a nonnegative $P_{B}$-supermartingale, hence $P_{B}$-a.e. convergent and therefore bounded in $n P_{B}$-a.e., giving (3.6). Due to (3.5) and (3.7), (3.8) then follows immediately from Hunt's lemma; see [3], V.45.

4. The main result and its proof. We are now ready to prove our main result, recalled here for convenience.

THEOREM 1.6. Suppose the $\mathbb{R}^{d}$-valued semimartingale $X$ satisfies the structure condition $(S C)$ and let $H$ be a payment stream. If the mean-variance tradeoff process $K=$ $\int \lambda^{\operatorname{tr}} d\langle M\rangle \lambda$ (or, equivalently, $A$ ) is continuous, the following are equivalent for an $L^{2}$ strategy $\varphi$ :

1) $\varphi$ is locally risk-minimizing for $H$.

2) $\varphi$ is 0-achieving and mean-self-financing, and the cost process $C^{H}(\varphi)$ is strongly orthogonal to $M$.

In particular, the concept "locally risk-minimizing" does not depend on the choice of B.

Proof. a) Thanks to Proposition 2.1, we can in both cases assume that $\varphi$ is 0 -achieving and mean-self-financing. Fix an increasing sequence $\left(\tau_{n}\right)_{n \in \mathbb{N}}$ of partitions of $[0, T]$ tending to the identity and take a small perturbation $\Delta=(\delta, \varepsilon)$. By Lemma 1.7, we can (and later shall) choose $\Delta$ such that $\varepsilon_{t}=0$ for all $t<T$. Now apply Proposition 2.2 to write for each $n \in \mathbb{N}$, using the shorthand $A_{i}^{n}$ for $A_{i}^{\tau_{n}}$, that

$$
r^{\tau_{n}}[\varphi, \Delta ; H]=A_{1}^{n}+A_{2}^{n}+A_{3}^{n}+A_{4}^{n} .
$$

We want to argue next that $A_{2}^{n}, A_{3}^{n}$ and $A_{4}^{n}$ can all be neglected asymptotically. 
b) Let $U:=\int|\delta d A|=\int\left|\delta^{\operatorname{tr}} \sigma \lambda\right| d B$ denote the variation of $\int \delta d A$. For $t \leq t^{\prime}$, we then have $\left|\int_{t}^{t^{\prime}} \delta_{s} d A_{s}\right| \leq U_{t^{\prime}}-U_{t}$, and the explicit expression for $A_{2}^{\tau_{n}}$ in Proposition 2.2 gives

$$
\begin{aligned}
A_{2}^{n} & =\sum_{t_{i}, t_{i+1} \in \tau_{n}} \frac{\operatorname{Var}\left[\int_{t_{i}}^{t_{i+1}} \delta_{s} d A_{s} \mid \mathcal{F}_{t_{i}}\right]}{E\left[B_{t_{i+1}}-B_{t_{i}} \mid \mathcal{F}_{t_{i}}\right]} I_{\left(t_{i}, t_{i+1}\right]} \\
& \leq \sum_{t_{i}, t_{i+1} \in \tau_{n}} \frac{E\left[\left(U_{t_{i+1}}-U_{t_{i}}\right)^{2} \mid \mathcal{F}_{t_{i}}\right]}{E\left[B_{t_{i+1}}-B_{t_{i}} \mid \mathcal{F}_{t_{i}}\right]} I_{\left(t_{i}, t_{i+1}\right]} \\
& =\tilde{Q}_{2}\left[U, \tau_{n}\right] .
\end{aligned}
$$

Next use Cauchy-Schwarz and the notation $Y:=\left\langle\int \delta d M\right\rangle+\left\langle C^{H}(\varphi)\right\rangle$ to get

$$
\begin{aligned}
& \left|\operatorname{Cov}\left(\int_{t_{i}}^{t_{i+1}} \delta_{s} d M_{s}-\left(C_{t_{i+1}}^{H}(\varphi)-C_{t_{i}}^{H}(\varphi)\right), \int_{t_{i}}^{t_{i+1}} \delta_{s} d A_{s} \mid \mathcal{F}_{t_{i}}\right)\right|^{2} \\
\leq & E\left[\left(\int_{t_{i}}^{t_{i+1}} \delta_{s} d M_{s}-\left(C_{t_{i+1}}^{H}(\varphi)-C_{t_{i}}^{H}(\varphi)\right)\right)^{2} \mid \mathcal{F}_{t_{i}}\right] E\left[\left(\int_{t_{i}}^{t_{i+1}} \delta_{s} d A_{s}\right)^{2} \mid \mathcal{F}_{t_{i}}\right] \\
\leq & 2 E\left[Y_{t_{i+1}}-Y_{t_{i}} \mid \mathcal{F}_{t_{i}}\right] E\left[\left(U_{t_{i+1}}-U_{t_{i}}\right)^{2} \mid \mathcal{F}_{t_{i}}\right] .
\end{aligned}
$$

Combining this with the expression for $A_{3}^{\tau_{n}}$ in Proposition 2.2 and Cauchy-Schwarz yields

$$
\begin{aligned}
\left|A_{3}^{n}\right| \leq & \sqrt{8}\left(\sum_{t_{i}, t_{i+1} \in \tau_{n}} \frac{E\left[Y_{t_{i+1}}-Y_{t_{i}} \mid \mathcal{F}_{t_{i}}\right]}{E\left[B_{t_{i+1}}-B_{t_{i}} \mid \mathcal{F}_{t_{i}}\right]} I_{\left(t_{i}, t_{i+1}\right]}\right)^{\frac{1}{2}} \\
& \times\left(\sum_{t_{i}, t_{i+1} \in \tau_{n}} \frac{E\left[\left(U_{t_{i+1}}-U_{t_{i}}\right)^{2} \mid \mathcal{F}_{t_{i}}\right]}{E\left[B_{t_{i+1}}-B_{t_{i}} \mid \mathcal{F}_{t_{i}}\right]} I_{\left(t_{i}, t_{i+1}\right]}\right)^{\frac{1}{2}} \\
= & \sqrt{8}\left(\tilde{Q}_{1}\left[Y, \tau_{n}\right]\right)^{\frac{1}{2}}\left(\tilde{Q}_{2}\left[U, \tau_{n}\right]\right)^{\frac{1}{2}} .
\end{aligned}
$$

Now $\left\langle\int \delta d M\right\rangle$ is bounded and $C^{H}(\varphi)$ is a square-integrable martingale; therefore $Y$ is increasing and integrable so that $\tilde{Q}_{1}\left[Y, \tau_{n}\right]=\left.\frac{d P_{Y}}{d P_{B}}\right|_{\mathcal{P}^{\tau_{n}}}$ by Lemma 3.4. So $\left(\tilde{Q}_{1}\left[Y, \tau_{n}\right]\right)_{n \in \mathbb{N}}$ is a nonnegative $P_{B}$-supermartingale, thus convergent and hence bounded in $n P_{B}$-a.e.

Finally, the term $A_{4}^{n}$ is by Proposition 2.2 always nonnegative. If the small perturbation $\Delta=(\delta, \varepsilon)$ has $\varepsilon_{t}=0$ for all $t<T$, the explicit expression for $A_{4}^{\tau_{n}}$ gives

$$
\begin{aligned}
A_{4}^{n} & =\sum_{t_{i}, t_{i+1} \in \tau_{n}} \frac{\left(E\left[\int_{t_{i}}^{t_{i+1}} \delta_{s} d A_{s} \mid \mathcal{F}_{t_{i}}\right]\right)^{2}}{E\left[B_{t_{i+1}}-B_{t_{i}} \mid \mathcal{F}_{t_{i}}\right]} I_{\left(t_{i}, t_{i+1}\right]} \\
& \leq \sum_{t_{i}, t_{i+1} \in \tau_{n}} \frac{E\left[\left(U_{t_{i+1}}-U_{t_{i}}\right)^{2} \mid \mathcal{F}_{t_{i}}\right]}{E\left[B_{t_{i+1}}-B_{t_{i}} \mid \mathcal{F}_{t_{i}}\right]} I_{\left(t_{i}, t_{i+1}\right]}=\tilde{Q}_{2}\left[U, \tau_{n}\right] .
\end{aligned}
$$

Thus controlling $\tilde{Q}_{2}\left[U, \tau_{n}\right]$ is the key to understanding the asymptotics of $r^{\tau_{n}}[\varphi, \Delta ; H]$.

c) We show below in step d) that

$$
\lim _{n \rightarrow \infty} \tilde{Q}_{2}\left[U, \tau_{n}\right]=0 \quad P_{B} \text {-a.e. }
$$

Accepting this for the moment, let us prove the equivalence of 1) and 2). If $\varphi$ is locally risk-minimizing, then $\liminf _{n \rightarrow \infty} r^{\tau_{n}}[\varphi, \Delta ; H] \geq 0 P_{B}$-a.e. for every small perturbation $\Delta=(\delta, \varepsilon)$. If we choose a $\Delta$ such that $\varepsilon_{t}=0$ for all $t<T,(4.2)$ and the estimates in 
step b) imply

$$
\lim _{n \rightarrow \infty} A_{i}^{n}=0 \quad P_{B} \text {-a.e. for } i=2,3,4,
$$

and we know from Lemma 3.1 that

$$
\lim _{n \rightarrow \infty} A_{1}^{n}=\left(\delta-2 \mu^{H}\right)^{\operatorname{tr}} \sigma \delta \quad P_{B} \text {-a.e. }
$$

Hence we obtain from (4.1) that $\left(\delta-2 \mu^{H}\right)^{\operatorname{tr}} \sigma \delta \geq 0 P_{B^{-a}}$.e. for every $\delta$ such that $\left\langle\int \delta d M\right\rangle$ and $\left|\delta^{\operatorname{tr}} \sigma \lambda\right|$ are bounded, and thus $\left(\mu^{H}\right)^{\operatorname{tr}} \sigma \mu^{H}=0 P_{B^{-a}}$ a.e. by Proposition 3.2. This shows that $C^{H}(\varphi)$ is strongly orthogonal to $M$.

Conversely, suppose that $C^{H}(\varphi)$ is strongly orthogonal to $M$ so that $\left(\mu^{H}\right)^{\operatorname{tr}} \sigma \mu^{H}=0$ $P_{B}$-a.e. and thus, again by Proposition 3.2, $\left(\delta-2 \mu^{H}\right)^{\operatorname{tr}} \sigma \delta \geq 0 P_{B}$-a.e. for every $\delta$ such that $(\delta, \varepsilon)$ is a small perturbation for some $\varepsilon$. By (4.2) and the estimates in step b),

$$
\lim _{n \rightarrow \infty} A_{i}^{n}=0 \quad P_{B} \text {-a.e. for } i=2,3,
$$

and $\lim \inf _{n \rightarrow \infty} A_{4}^{n} \geq 0 P_{B}$-a.e. since $A_{4}^{n} \geq 0$. Combining this with (4.1) and (4.3) yields for every small perturbation $\Delta$ that $\liminf _{n \rightarrow \infty} r^{\tau_{n}}[\varphi, \Delta ; H] \geq 0 P_{B}$-a.e., and so $\varphi$ is locally risk-minimizing.

d) It remains to prove (4.2). Since $\delta$ comes from a small perturbation and $B$ is bounded, both $\left|\delta^{\operatorname{tr}} \sigma \lambda\right|$ and $U=\int\left|\delta^{\operatorname{tr}} \sigma \lambda\right| d B=\int|\delta d A|$ are bounded as well. Moreover, $U$ is continuous (because $K=\int \lambda^{\mathrm{tr}} \sigma \lambda d B$ is so, by assumption) and null at 0 with bounded 1-variation so that Proposition 3.5 with $r=1$ and $p=2$ yields $\lim _{n \rightarrow \infty} Q_{2}\left[U, \tau_{n}\right]=0$ $P_{B}$-a.e. Hence (4.2) will follow from Proposition 3.5 once we prove that

$$
\sup _{n \in \mathbb{N}} Q_{2}\left[U, \tau_{n}\right] \in L^{1}\left(P_{B}\right) .
$$

But since $U$ is increasing and $d P_{U} / d P_{B}=\left|\delta^{\text {tr }} \sigma \lambda\right|$, we get from Lemma 3.4

$$
\begin{aligned}
Q_{2}\left[U, \tau_{n}\right] & =\sum_{t_{i}, t_{i+1} \in \tau_{n}} \frac{\left(U_{t_{i+1}}-U_{t_{i}}\right)^{2}}{B_{t_{i+1}}-B_{t_{i}}} I_{\left(t_{i}, t_{i+1}\right]} \\
& \leq U_{T} Q_{1}\left[U, \tau_{n}\right]=\left.U_{T} \frac{d P_{U}}{d P_{B}}\right|_{\mathcal{O}^{\tau_{n}}}=U_{T} E_{B}\left[\left|\delta^{\operatorname{tr}} \sigma \lambda\right| \mid \mathcal{O}^{\tau_{n}}\right],
\end{aligned}
$$

and because $U_{T}$ and $\left|\delta^{\text {tr }} \sigma \lambda\right|$ are both bounded by some constant, so is $Q_{2}\left[U, \tau_{n}\right]$, uniformly in $n$. This gives (4.4) and thus completes the proof.

Apart from providing a streamlined exposition, the results in this paper extend earlier work on local risk-minimization in three directions:

1) We treat payment streams $\left(H_{t}\right)_{0 \leq t \leq T}$ instead of European contingent claims $H_{T}$ due at time $T$, thus extending to the general semimartingale setting work done by [7] for the case where $X$ is a martingale. This has independently also been done in [1]. However, we point out that passing from $H_{T}$ to $\left(H_{t}\right)$ is quite simple and constitutes no major contribution, as will again become apparent in the next section.

2) We treat a multidimensional setting with $d \geq 1$ risky assets by allowing $X$ to be $\mathbb{R}^{d}$-valued. The feasibility of this extension was announced in [13], but the work has not been done in the literature so far. While technically not very difficult, it 
needs a careful formulation and treatment, and we view this as one of our two main contributions.

3) We remove several technical conditions on the underlying price process $X$; only the structure condition (SC) and continuity of $A$ are required, thanks to the improved formulation of the basic criterion. This is our second main contribution.

To emphasize the improvements made here, we briefly look at the classical case from [11] where $X$ is one-dimensional and only a European contingent claim $H_{T} \in L^{2}\left(P, \mathcal{F}_{T}\right)$ is considered. In comparison with [11], we no longer need the assumption (X2) that $\langle M\rangle$ is $P$-a.s. strictly increasing on $[0, T]$, nor (X5) that $X$ is $P$-a.s. continuous at $T$, nor any global integrability on $A$ or $\lambda$ as in (X4). We work with a smaller (more restrictive) class of small perturbations than in [11]; but the equivalent characterization of local risk-minimality via part 2) of Theorem 1.6 is the same as in Proposition 2.3 of [11], and so our approach here is equivalent to the one in [11]. In particular, this also shows that

(4.5) the notion of pseudo-optimality introduced in [13] for an $L^{2}$-strategy coincides with local risk-minimality.

5. A simple application. To round off the paper, we present in this section the link between local risk-minimization and the Föllmer-Schweizer decomposition. This is quite simple and well known and only done for completeness. We use the same setup as in Section 1.

Definition 5.1. An $\mathcal{F}_{T}$-measurable random variable $Y \in L^{2}(P)$ admits a FöllmerSchweizer decomposition if it can be written as

$$
Y=Y^{(0)}+\int_{0}^{T} \vartheta_{s}^{Y} d X_{s}+L_{T}^{Y} \quad P \text {-a.s. }
$$

where $Y^{(0)} \in L^{2}(P)$ is $\mathcal{F}_{0}$-measurable, $\vartheta^{Y}$ is in $\Theta_{S}$, and the process $L^{Y}=\left(L_{t}^{Y}\right)$ is a (right-continuous) square-integrable martingale null at 0 and strongly orthogonal to $M$.

Thanks to Theorem 1.6, we obtain

Proposition 5.2. Suppose the $\mathbb{R}^{d}$-valued semimartingale $X$ satisfies the structure condition (SC) and the mean-variance tradeoff process $K=\int \lambda^{\operatorname{tr}} d\langle M\rangle \lambda$ (or, equivalently, A) is continuous. Then a payment stream $H$ admits a locally risk-minimizing $L^{2}$-strategy $\varphi$ if and only if $H_{T}$ admits a Föllmer-Schweizer decomposition. In that case, $\varphi=(\vartheta, \eta)$ is given by

$$
\vartheta=\vartheta^{H_{T}}, \quad \eta=V^{H_{T}}-\left(\vartheta^{H_{T}}\right)^{\operatorname{tr}} X
$$

with

$$
V_{t}^{H_{T}}:=H_{T}^{(0)}+\int_{0}^{t} \vartheta_{s}^{H_{T}} d X_{s}+L_{t}^{H_{T}}-H_{t}, \quad 0 \leq t \leq T,
$$

and then

$$
C_{t}^{H}(\varphi)=H_{T}^{(0)}+L_{t}^{H_{T}}, \quad 0 \leq t \leq T .
$$

Proof. If $H_{T}$ has a Föllmer-Schweizer decomposition (5.1), then (5.2) and (5.3) define an $L^{2}$-strategy $\varphi$ whose cost process is given by (5.4). Hence $\varphi$ is mean-self-financing 
and also 0-achieving by (5.1) and thus locally risk-minimizing for $H$ by Theorem 1.6. Conversely, if $\varphi=(\vartheta, \eta)$ is locally risk-minimizing for $H$, we can write the condition $V_{T}(\varphi)=0$ as

$$
H_{T}=C_{T}^{H}(\varphi)+\int_{0}^{T} \vartheta_{s} d X_{s}=C_{0}^{H}(\varphi)+\int_{0}^{T} \vartheta_{s} d X_{s}+\left(C_{T}^{H}(\varphi)-C_{0}^{H}(\varphi)\right),
$$

and so we have (5.1) for $H_{T}$ with

$$
H_{T}^{(0)}:=C_{0}^{H}(\varphi), \quad \vartheta^{H_{T}}:=\vartheta, \quad L^{H_{T}}:=C^{H}(\varphi)-C_{0}^{H}(\varphi) ;
$$

note that $L^{H_{T}}$ is a martingale and strongly orthogonal to $M$ by Theorem 1.6.

Proposition 5.2 is a slight generalization (to payment streams) of Proposition (2.24) in [4]; see also Proposition 3.4 in [13]. Apart from subtracting the process $H$ in (5.3), the proof remains unchanged. It is interesting to note that the extension from European contingent claims to payment streams involves no difficulties at all and that the key quantity to examine is only the total payment $H_{T}$. This is due to the fact that our strategies need not be self-financing, so that any intermediate payments can simply be added to the costs.

REMARK 5.3. Proposition 5.2 gives a link between local risk-minimization and the Föllmer-Schweizer decomposition which holds true in full generality. Finding the FöllmerSchweizer decomposition, however, is not always easy. It has been shown in [4] and [13] that (up to some mild integrability conditions) this decomposition can be obtained as the Galtchouk-Kunita-Watanabe (GKW) decomposition under the so-called minimal martingale measure $\widehat{P}$, if $X$ is continuous. For discontinuous $X$, the result in Proposition 5.2 is still true, but using the GKW decomposition under $\widehat{P}$ may fail to give the correct decomposition. A more detailed discussion of this issue is given in [14], whose authors in particular point out and improve upon some incorrect work in the literature.

\section{References}

[1] J. Barbarin, Local risk-minimization for payment processes. Application to insurance contracts with surrender option or to default sensitive contingent claims, working paper, Université Catholique de Louvain, 2007.

[2] F. Delbaen and W. Schachermayer, The existence of absolutely continuous local martingale measures, Annals of Applied Probability 5 (1995), 926-945.

[3] C. Dellacherie and P. A. Meyer, Probabilities and Potential B, North-Holland, Amsterdam, 1982.

[4] H. Föllmer and M. Schweizer, Hedging of contingent claims under incomplete information, in: Applied Stochastic Analysis, M. H. A. Davis and R. J. Elliott (eds.), Stochastics Monographs, Gordon and Breach, London, 1991, 389-414.

[5] H. Föllmer and D. Sondermann, Hedging of non-redundant contingent claims, in: Contributions to Mathematical Economics, W. Hildenbrand and A. Mas-Colell (eds.), NorthHolland, Amsterdam, 1986, 205-223.

[6] J. M. Harrison and S. R. Pliska, Martingales and stochastic integrals in the theory of continuous trading, Stochastic Processes and their Applications 11 (1981), 215-260. 
[7] T. Møller, Risk-minimizing hedging strategies for insurance payment processes, Finance and Stochastics 5 (2001), 419-446.

[8] M. Riesner, Locally risk-minimizing hedging of insurance payment streams, ASTIN Bulletin 37 (2007), 67-92.

[9] M. Schweizer, Hedging of options in a general semimartingale model, Diss. ETH Zürich 8615, 1988.

[10] M. Schweizer, Risk-minimality and orthogonality of martingales, Stochastics and Stochastics Reports 30 (1990), 123-131.

[11] M. Schweizer, Option hedging for semimartingales, Stochastic Processes and their Applications 37 (1991), 339-363.

[12] M. Schweizer, On the minimal martingale measure and the Föllmer-Schweizer decomposition, Stochastic Analysis and Applications 13 (1995), 573-599.

[13] M. Schweizer, A guided tour through quadratic hedging approaches, in: Option Pricing, Interest Rates and Risk Management, E. Jouini, J. Cvitanić and M. Musiela (eds.), Cambridge University Press, Cambridge, 2001, 538-574.

[14] N. Vandaele and M. Vanmaele, A locally risk-minimizing hedging strategy for unit-linked life insurance contracts in a Lévy process financial market, Insurance: Mathematics \& Economics 42 (2008), 1128-1137. 
